which are not recommended before 1 year: fruity juice, sweet drinks, honey, sugar, salt, tea. Many parents (40,5\%) switched on TV (video) while feeding. Some of them $(14,9 \%)$ forced the baby to eat whole portion of food. The child's appetite was considered as satisfactory in $91,7 \%$ of families. In $1 / 3$ families the food-pieces chewing skills and self 'finger-food' eating skills were not developed.

The totally $11,9 \%$ of children had an overweight and thus risk of obesity in the age of 1 year according to weight/length chart. The possible reasons for overweight were increased part of easily-absorbed carbohydrates in the diet and non-responsive feeding which lead to overconsumption of food by children.

\section{NEUROLOGICAL FINDINGS OF VITAMIN B12 DEFICIENCY IN INFANCY}

Ayșenur Levent*, Yelda Türkmenoğlu, Tuğçe Aksu Uzunhan, Emine Türkkan, Adem Karbuz. Okmeydani Research and Training Hospital

\subsection{6/archdischild-2021-europaediatrics.42}

Vitamin B12 is essential for the development of the central nervous system.

The lack of the vitamin B12 leads to neurological symptoms developing more rapidly in the first year of life. The most common neurologic symptoms observed during infancy are apathy, developmental delay or retardation, involuntary movements, hypotonia, and seizures. In our study, the objective was to determine the neurological symptoms and signs of vitamin B12 deficiency in children and to emphasize the consequences of early diagnosis and treatment.

A retrospective study was performed over the period 20172020 at a third level research and training hospital in Turkey. We studied 43 infants aged 1 to 24 months with vitamin B12 deficiency who were hospitalized and treated for other diseases in the paediatrics department of the hospital. The diagnosis of vitamin B12 deficiency was based on haematological values, a low serum vitamin B12 level, a normal level of folic acid, and a high serum homocysteine levels. Complete blood count, serum vitamin B12, folic acid, ferritin, homocysteine, NH3, and lactate dehydrogenase levels were measured in all patients, along with serum vitamin B12 levels in their mothers.

The mean age of 27 male and 16 female patients was

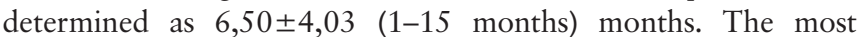
common symptom was afebrile seizures. This was followed by weakness and tremor. Optic atrophy was detected on the ophthalmic examination of one of the patients with convulsions. On neurologic examination, the most common finding was apathy. Hypotonia, involuntary movements and lack of eye contact were also observed.

Retrospectively extra neurologic findings were observed in 25 patients. Most of these had pallor (11), 5 had failure to thrive, 5 had hyperpigmentation of skin, 4 had glossitis, and 1 had jaundice. Anaemia was determined in 37,2\% of the cases. Bicytopenia was determined in $9.3 \%$ of patients, thrombocytopenia in $4.7 \%$, and pancytopenia was found in $2.3 \%$ of patients.

In conclusion, vitamin B12 is important for development of the brain and nutritional deficiencies are common, especially in developing countries.

Most of them occur in exclusively breast-fed infants of deficient mothers.
Even when laboratory parameters are all within normal values, the clinical condition should encourage us to research B12 deficiency. Early recognition of these infants is important because this condition is partially reversible and can aid in preventing the progression of irreversible deficits. More importantly, vitamin B12 supplementation of pregnant women may help prevent neurological findings in infants.

\section{EXPERIENCE OF THE USE OF HERBAL MEDICINAL PRODUCT TONSILGON N IN PRESCHOOL CHILDREN WITH WALDEYER'S TONSILLAR RING MEDICAL CONDITION}

Vera Vavilova*, Aleksandr M Vavilov, Asya A Cherkayeva, Irina A Nechayeva, Kirill K Bessonov. Kemerovo State Medical University

\subsection{6/archdischild-2021-europaediatrics.43}

Study objective: to evaluate efficacy and safety of the use of the herbal medicinal product Tonsilgon $\mathrm{N}$ (HMP) in clinical practice in preschool children with Waldeyer's tonsillar ring medical condition.

Materials and Methods 628 children (aged 2-5 years, median age $-3.34 \pm 2.78$, girls -316 (50.4\%), boys - 312 (49.6\%)) with chronic conditions of the pharyngeal and palatine tonsils were enrolled in the study. All children were divided into two comparable groups. Children in group I $(n=317)$ received HMP in drops during 60 days in overall.

Preschoolers in the group II $(n=311)$ did not receive HMP. Clinical efficacy and safety evaluation of the drug was performed before and after the treatment course.

Results HMP therapy (group I) eliminated the signs of adenoiditis and led to a decrease in the number of children with second degree tonsil hypertrophy from $75.0-6.84 \%$ to $36.1-$ $4.62 \%$ ( $\mathrm{p}<0.001)$. Correspondingly, the number of children with I degree hypertrophy of pharyngeal tonsils increased $(p<0.01)$. Adenoiditis signs and symptoms were almost resolved in $75.8 \%-5.34 \%$ of patients; symptomatic treatment of adenoiditis was not effective $(p>0.05)$ The degree of hypertrophy of pharyngeal and palatine tonsils in preschoolers of the 2nd group did not change and even showed a tendency to increase to $83.6 \%$.

By the end of HMP therapy, children in group I demonstrated a good overall well-being, the swelling of palatine tonsil subsided, adequate nasal breathing was restored, there was no pathological discharge from the pharyngeal tonsils. One year after the end of treatment, nasal breathing was restored in $62.7 \%$ of patients who received HMP $(p<0.01)$; snoring stopped in $81.8 \%$ of patients $(\mathrm{p}<0.01)$. Treatment with HMP was accompanied by an increase in the level of lysozyme in nasal secretions from $56.9-0.88 \%$ to $69.8-0.45 \% \quad(\mathrm{p}<0.001)$ and SIgA from $0.18-0.005 \mathrm{~g} / \mathrm{L}$ to $0.20-0.003 \mathrm{~g} / \mathrm{L}(\mathrm{p}<0.01)$. In group II, no significant change was observed in the indicators characterising the state of local immunity. Among those who received HMP, 90\% showed excellent and good results. Symptomatic treatment in group II did not affect the size of the pharyngeal tonsil and local immunity parameters.

Conclusions Study results confirmed efficacy and good tolerance of the HMP.

Evaluation of the frequency of exacerbations of chronic ENT pathology in children who received this HMP during rehabilitation confirmed its effectiveness in boosting of respiratory tract immunity. 